\begin{tabular}{lllllllllllllllllllllllllllllllll}
\hline$R$ & $E$ & $V$ & I & S & T & A & D & E & E & S & T & U & D & I & O & S & I & N & T & E & $R$ & N & A & C & I & O & N & $A$ & A & $E$ & $S$
\end{tabular}

\title{
Los acuerdos comerciales en el Asia-Pacífico de cara a los objetivos de APEC
}

\author{
Robert Scollay y Fernando González-Vigil ${ }^{*}$
}

\begin{abstract}
El trabajo plantea la necesidad de que las economías miembros de APEC establezcan una acción coordinada destinada a asegurar que la reciente proliferación de acuerdos comerciales preferenciales en el Asia-Pacifico contribuya al logro del objetivo de que APEC en su conjunto sea una región de comercio e inversión libres en el año 2010, para el caso de las economías desarrolladas, y en el 2020 para las que están en desarrollo. Los autores sustentan este planteamiento comparando la liberalización no discriminatoria promovida por APEC con la liberalización preferencial pactada en los mencionados acuerdos, examinando los problemas que esta última acarrea para la extensión de la liberalización a escalas más amplias, y analizando los resultados de las principales investigaciones empíricas que muestran inequívocamente que los beneficios económicos de la primera son mayores.
\end{abstract}

\section{APEC: Metas de Bogor y ENFoque DE LIBERALIZACIÓN REGIONAL}

n la Declaración de Bogor (1994), los miembros del APEC se fijaron metas precisas para realizar su visión del Asia-Pacífico como una región de libre comercio e inversión, comprometiéndose a implementar las medidas de liberalización y facilitación necesarias para que esa visión sea realidad en 2010 en el caso de las economías desarrolladas, y en 2020 en el de aquéllas en desarrollo.

* Este artículo es una traducción parcial del documento "Asia Pacific RTAs as Avenues for Achieving APEC's Bogor Goals”, PECC, septiembre 2003 (disponible en: www.pecc.org/trade), donde se sintetizan los principales resultados de las labores sobre ACRs realizadas por el mencionado foro en el período 2001-2003. 
Estas metas de Bogor deben alcanzarse mediante la aplicación de un enfoque de liberalización regional singular al APEC y cuyos elementos distintivos más importantes son los siguientes: que el proceso de liberalización regional no sea discriminatorio, rasgo clave éste del "regionalismo abierto" del APEC ${ }^{1}$, que contrasta con la discriminación inherente a los acuerdos comerciales regionales tradicionales y que deja en claro que APEC no pretende ser un bloque regional convencional; que las metas deben lograrse de un modo que contribuya a la creciente apertura y fortaleza del sistema multilateral de comercio; y que el APEC fue explícitamente concebido como una agrupación regional que comprende ambos lados del Océano Pacífico, es decir, como una entidad transpacífica.

\section{Las metas de Bogor deben alcanzarse aplicando un enfoque de la liberalización regional propio del APEC.}

Para los miembros de APEC, la adopción del compromiso de Bogor fue una consecuencia lógica de la constatación de que su exitoso crecimiento y desarrollo económico estaban basados tanto en la apertura y estabilidad de los mercados global y regional como en los fuertes vínculos de negocios, comercio e inversión ya establecidos a lo largo de su ámbito, entre ambos lados del Océano Pacífico (dimensión transpacífica), entre las economías del Pacífico occidental (dimensión intra-Pacífico occidental) y entre las economías de las Américas (dimensión intra-americana).

Ese compromiso también reflejó el convencimiento de que las principales beneficiarias de la liberalización del comercio y de la inversión son las propias economías que la emprenden. Siendo esto especialmente cierto para el caso de las economías pequeñas, la resultante racionalidad económica en pro de la liberalización unilateral reconoce que sus beneficios económicos serán mayores si todos los socios comerciales la emprenden al mismo tiempo, ya que para las economías grandes la simultaneidad de la liberalización unilateral puede ser esencial para asegurarse su parte de dichos beneficios. De ahí el término "unilateralismo concertado", acuñado en APEC para tipificar la modalidad básica de su proceso de liberalización regional.

Asimismo, los miembros del APEC entienden que las economías en desarrollo necesitan construir la capacidad humana e institucional que les permita aprovechar óptimamente los beneficios potenciales de la liberalización y asegurarse de que ésta contribuya efectivamente a sus objetivos de desarrollo; reconocen también que para tal aprovechamiento óptimo se requieren reformas económicas

${ }^{1}$ APEC dejó sin resolver la cuestión de si la no discriminación se aplica de manera incondicional o si depende de la reciprocidad, permitiendo así que, de una u otra manera, cada economía miembro adhiera al principio de "regionalismo abierto", quizás debido a que esta forma de aplicar el principio de flexibilidad del APEC parecía suficiente en una época en que no estaban proliferando los acuerdos comerciales preferenciales entre los miembros de ese foro, como sucedía en la época de Bogor. 
básicas de la estructura de los mercados y de la administraciones pública y empresarial. De ahí, por un lado, la insistencia en que en la agenda de APEC, la cooperación económica y técnica ocupe un lugar igual que el de la liberalización y facilitación del comercio y la inversión y, por el otro, la inclusión en la Osaka Action Agenda (1995) de temas como la política de competencia y reformas en la regulación de los mercados, que han sido aún más enfatizados a resultas de la crisis económica del este de Asia en 1997-1998.

\section{Las beneficiarias de la liberalización del comercio y de la inversión son las economías que la emprenden.}

EVOLUCIÓN DE LOS ACUERDOS

COMERCIALES REGIONALES

en El AsIA-PACífICO

Cuando en 1994 se emitió la Declaración de Bogor, existían entre grupos de economías miembros del APEC tres acuerdos comerciales regionales (ACR) de tipo convencional, en el sentido de practicantes de una liberalización discriminatoria: el NAFTA, el AFTA (ASEAN Free Trade Agreement) y el ANZCERTA (Australia-New Zealand Closer Economic Relations Trade Agreement). A ellos se sumarían luego los dos acuerdos de libre comercio (ALC) bilaterales suscritos por Chile con sendos miembros del NAFTA, Canadá y México, en 1996 y 1998, respectivamente; en tanto que por esos años no llegó a concretarse el propuesto ALC bilateral entre Estados Unidos y ese país sudamericano.

Fue a partir de 1998 que empezaron a proliferar las iniciativas de concertación de ACR entre miembros del APEC. A la fecha, seis de éstos ya han producido acuerdos (Singapur-Japón, Singapur-Estados Unidos, Chile-Estados Unidos, Chile-Corea, Singapur-Nueva Zelandia, y Singapur-Australia), pero varias otras se encuentran en distintos estadios de negociación, estudio o discusión. La gran mayoría de las iniciativas de esta nueva "ola" de ACR en el Asia-Pacífico son de carácter bilateral, y generalmente involucran asociaciones entre una economía pequeña o mediana y uno de los tres gigantes económicos de la región (por ejemplo, las tres primeras antes mencionadas, más Australia-Estados Unidos, Corea-Japón, México-Japón, Tailandia-Japón, Hong Kong-China) o con otra economía pequeña o mediana (por ejemplo, las otras tres ya mencionadas, más Singapur-Canadá, Singapur-México, Singapur-Corea, México-Corea, Tailandia-Australia, Hong Kong-Nueva Zelandia). También ha habido ocasionalmente algunas iniciativas de ACR plurilaterales, como el "P5" (entre Estados Unidos, Australia, Singapur, Chile y Nueva Zelandia) informalmente propuesto hace unos años, o la actual propuesta de un ALC trilateral entre Singapur, Nueva Zelandia y Chile.

Destaca el hecho de que un gran número de estas recientes iniciativas bilaterales y plurilaterales sean transpacíficas. Respecto de aquéllas entre socios de un solo lado del Océano Pacífico, en su lito- 
ral occidental se han establecido tanto al interior del este de Asia como entre economías de esa subregión y de Oceanía, mientras que al interior de las Américas casi todos los flujos comerciales entre los miembros de APEC están ya cubiertos por ACR, y estas economías participan actualmente tanto en nuevas iniciativas de ACR bilaterales o plurilaterales con países de su hemisferio como en las negociaciones para la creación de un gran bloque comercial americano: el ALCA.

La posibilidad de que también en el este de Asia se establezca un gran bloque comercial surgió con la propuesta de celebrar un ALC a nivel de la agrupación "ASEAN + 3" (compuesta por las diez economías de ASEAN más China, Corea y Japón), originalmente establecida con fines de cooperación monetaria a raíz de la crisis asiática. De concretarse esta posibilidad, así como la creación del ALCA, el escenario emergente podría ser el de un "Pacífico bipolar", dividido entre los dos "megabloques" en ambos lados de ese océano y, si se adiciona a la Unión Europea, el escenario global podría ser el de un "sistema comercial tripolar", basado en tres "megabloques" que en conjunto representarían aproximadamente un $90 \%$ del PIB mundial.

Sin embargo, parece que la propuesta de un ALC “ASEAN + 3" se está eclipsando por más recientes iniciativas de formato "ASEAN + 1". Éstas empezaron con la propuesta china de negociar un ALC China-ASEAN, a la que Japón inmediatamente respondió proponiendo un acuerdo tipo CEP (Closer Economic Partnership) entre ese país y ASEAN, que potencialmente incluiría un ALC. Luego los Estados Unidos lanzaron su "iniciativa para ASEAN", que comprende un acuerdo marco con ASEAN como grupo y la negociación de ALC bilaterales con algunos de los miembros de esa subregión.

\section{La mayoría de los nuevos acuerdos comerciales regionales suscritos en Asia-Pacífico son de carácter bilateral.}

Estas iniciativas, junto con otras bilaterales contempladas por Estados Unidos y por Japón, anuncian la posible configuración de un patrón de ACR tipo "Hub-and-Spoke" ("H\&S") en la región del Asia-Pacífico, como alternativa al "Pacífico bipolar". Este patrón implicaría el establecimiento de tres redes de ACR bilaterales centradas cada una en uno de los gigantes económicos de la región: Estados Unidos, Japón y China. Como todas esas redes involucran al sureste asiático, en ASEAN se ha planteado la necesidad de desplegar una estrategia subregional que le permita a esta agrupación posicionarse como " $h u b$ ". Para ello, además de los ya mencionados acuerdos con los tres gigantes económicos, recientemente se propuso un ALC ASEANIndia y desde hace ya buen tiempo está la propuesta de vincular al $A F T A$ con el $C E R$ de Australia y Nueva Zelandia, aunque los dos grupos siguen sin ponerse de acuerdo sobre si esta vinculación debe incluir o no la reducción arancelaria necesaria para arribar a un ALC AFTA-CER. 
Que ASEAN llegue o no a posicionarse como un "hub" alternativo depende crucialmente de que pueda o no mantener una posición unificada y negociar con terceros como un grupo cohesionado. Hay señales que indican que esto será difícil. Ante las anunciadas intenciones de Estados Unidos y de Japón de establecer ALC bilaterales con miembros individuales de ASEAN, algunos de estos ya se han mostrados dispuestos a seguir el camino bilateral emprendido por Singapur. Por ejemplo, Tailandia está en negociaciones tanto con Japón como con Australia. También se ha sugerido que el ALC China-ASEAN se transforme en la práctica en una serie de ALC bilaterales.

Los hechos parecen indicar, entonces, que la fuerza "gravitacional" ejercida por las grandes economías sobre los miembros individuales de ASEAN es mucho más poderosa que las aspiraciones de lograr que ASEAN como grupo sea un " $h u b$ " alternativo, y que prevalecerá en consecuencia una arquitectura " $H \& S$ " de corte tradicional, con las grandes economías en su papel tradicional de "hubs" y con los miembros individuales de ASEAN así como otras economías medianas o pequeñas de la región en su también tradicional papel de "spokes".

Los hechos parecen indicar, asimismo, que la arquitectura del patrón " $H \& S$ " de ACR que está formándose en el Asia-Pacífico probablemente será bastante compleja. En efecto, además de los tres potenciales "hubs" mayores o principales (Estados Unidos, China y Japón) y de las aspiraciones de ASEAN de ser un "hub" alternativo, algunas de las economías medianas o pequeñas de la región han implementado con éxito una serie de medidas, que incluyen una agresiva política de concertación de ALC bilaterales y plurilaterales, a fin de reforzar su condición de "hub secundario" (el caso de Singapur) o de posicionarse ventajosamente para lograr esa condición (los casos de Chile y México). Australia y Corea serían otros ejemplos de potenciales "hubs secundarios".

\section{Los acuerdos comerciales} preferenciales se distinguen del APEC, que aplica un enfoque de comercio abierto libre y no discriminatorio.

En todo caso, una característica común a todos estos ACR reside en su enfoque preferencial de liberalización comercial, y por ello es más riguroso denominarlos como acuerdos comerciales preferenciales (ACP), que discriminan en favor de sus miembros y, por tanto, en detrimento de los no miembros. Esta característica marca una nítida diferencia entre esos acuerdos y el APEC, cuyo enfoque de "regionalismo abierto" está incorporado en la visión de un flujo de comercio e inversión no discriminatorio y libre en la región del Asia-Pacífico, que orienta las metas de Bogor, como ya se dijo. Otro rasgo central de esa misma visión es la dimensión transpacífica de APEC, que también marca una diferencia clave con las iniciativas conducentes a un "Pacífico bipolar" o a una serie de configuraciones " $H \& S$ ” en la región. 
TEMAS Y PROBLEMAS DE LA

LIBERALIZACIÓN PREFERENCIAL

La potencial incompatibilidad de los ACP con el "regionalismo abierto" del APEC fue reconocida por sus líderes cuando formularon la Declaración de Bogor, razón por la que solicitaron al Eminent Persons Group (EPG) un informe sobre cómo resolver el asunto. Pero después de Bogor, éste no le dio urgencia debido al poco interés por crear ACP que había entonces en la región; en cambio, la proliferación de iniciativas de ACP surgidas en los últimos cinco años le han convertido en tema de primordial importancia.

\section{El supuesto de que algo de liberalización es mejor que nada no es necesariamente cierto.}

La teoría económica enseña que los ACP son una opción de segundo orden de importancia en materia de liberalización. Por su parte, los encargados de formular las políticas consideran que son una opción "pragmática", útil cuando la liberalización unilateral o multilateral no puede rendir los frutos deseados, sea por imperfecciones de los mercados o por consideraciones políticas o estratégicas. Algo de liberalización es mejor que nada, es el supuesto subyacente a tal pragmatismo, supuesto que el análisis económico muestra que no es necesariamente cierto, por lo que la cuestión clave consiste en identificar los requisitos que deben reunir los ACP para asegurar su contribución posi- tiva al bienestar nacional y mundial, así como una mayor apertura del sistema multilateral de comercio.

\section{CREACIÓN Y DESVIACIÓN DE COMERCIO}

Puesto que, por definición, los ACP discriminan contra los no miembros al establecer preferencias entre ellos, solamente una parte del incremento de comercio generado por un ACP será "creación de comercio" (CC) en sentido estricto, resultante de las nuevas oportunidades de intercambio eficiente abiertas por la reducción arancelaria entre los miembros. Otra parte de dicho incremento, en cambio, reflejará el reemplazo del comercio con no miembros por el comercio entre miembros, que goza de la preferencia arancelaria. Esta "desviación de comercio" (DC) claramente perjudica a los no miembros, cuyas exportaciones son así desplazadas, aunque este efecto negativo podría ser parcial o totalmente compensado por un potencial incremento de las importaciones de los miembros en caso de que su ACP acelerara su crecimiento económico. Además, esta desviación también puede perjudicar el bienestar económico de los propios miembros del ACP, en la medida en que importaciones más caras o de menor calidad desde ellos sustituyan a importaciones mejores desde los no miembros debido al margen preferencial convenido mediante el ACP. En definitiva, el ACP puede tener consecuencias negativas para sus miembros, si la DC es superior a la CC, o positivo, si ocurre lo contrario. Pero aun en este último caso, 
el efecto global en el bienestar mundial puede ser negativo, en caso de que el beneficio neto que el ACP reporta a sus miembros sea menor que el daño que ocasiona a los no miembros.

\section{La desviación del comercio puede perjudicar el bienestar económico de los miembros del acuerdo preferencial.}

Si bien estos distintos efectos generalmente se han analizado en relación con el comercio de bienes, la argumentación básica quizás sea aún más válida respecto del comercio de servicios. En efecto, el acceso a mercados es un objetivo importante para una economía exportadora de servicios y, al mismo tiempo, desde el punto de vista de una economía importadora de servicios, el objetivo clave es promover el ingreso de proveedores de servicios internacionalmente competitivos, que mejoren la eficiencia de su sector de servicios. Este objetivo puede verse perjudicado por una liberalización preferencial que otorgue ventajas a proveedores de servicios menos eficientes que sean parte del ACP.

\section{CReación Y DESVIACIÓN DE LAS INVERSIONES}

Los análisis coinciden en que atraer inversiones es una motivación central, de igual o incluso mayor prioridad que el estímulo del comercio, para el "nuevo regionalismo" que se ha producido desde la última década en varias partes del mun$\mathrm{do}^{2}$. Sin embargo, un ACP tiene efectos análogos en la inversión y en el comercio: además de creación de inversión, puede haber desviación de inversión en la medida en que el acceso preferencial al mercado ampliado del ACP atraiga hacia sus miembros inversiones que de no existir dicho acceso habría tenido lugar en economías no miembros. Parte de esta inversión desviada probablemente ha de ser un reflejo de la reacción de exportadores de los no miembros para contrarrestar su posible desplazamiento por competidores al interior del ACP.

\section{Patrones "HUB-AND-SPOKE"}

Son bien conocidas las desventajas para los "spokes" que conlleva la asimetría inherente al patrón " $H \& S$ " de los ACP: el " $h u b$ " puede aprovecharse de la competencia entre "spokes" y usar como precedentes los ACP suscritos con algunos para lograr similares o mayores concesiones al negociar con los demás; mientras que cada "spoke" sólo obtiene acceso al mercado del "hub", éste gana acceso a los mercados de todos los "spokes", lo que incrementa las ventajas del "hub" respecto de los "spokes" en materia de atracción de inversiones.

Una estrategia utilizada por los "spo-

${ }^{2}$ Devlin, R. y Estevadeordal, A., "What's new in the 'New Regionalism' in the Americas?", INTALITD-STA, Working Paper 6, Banco Interamericano de Desarrollo, Washington, DC, mayo de 2001. 
kes" para compensar total o parcialmente esas desventajas consiste en suscribir ACP entre ellos, especialmente entre los que mantienen una relación comercial significativa. Los "spokes" cuyas economías son exitosas pueden además valerse de la estrategia de asegurarse ACP con cada uno de los principales "hubs" y así, mediante la combinación de ambas estrategias, pueden no sólo contrarrestar las desventajas de ser "spoke" de un determinado "hub" principal, sino también obtener los beneficios de funcionar como un "hub secundario" dentro de su propia red de ACP con otros "spokes". Como se dijo, este tipo de configuraciones " $H \& S$ secundarias" están desplegándose en la región del Asia-Pacífico. En tal contexto, un "spoke" que no haya suscrito ningún ACP con un " $h u b$ " principal puede experimentar un importante perjuicio económico.

\section{GRANDES BLOQUES COMERCIALES}

La suscripción de grandes ACP promete mayores beneficios potenciales de eficiencia gracias al aumento de la competencia y a la explotación de economías de escala. Sin embargo, las disparidades entre sus miembros pueden ampliarse debido a la desigual capacidad de aprovechar esas oportunidades. Asimismo, estos ACP pueden mejorar significativamente la relación de intercambio de sus miembros, y por lo tanto, deteriorar de manera importante aquélla de los no miembros, y acicatear así las represalias de estos. La consiguiente posibilidad de que se desaten guerras comerciales es precisamente el temido escenario que explica los recelos que provoca un posible sistema de comercio mundial dominado por tres grandes bloques comerciales, especialmente si dos de ellos se aliaran contra el tercero.

\section{Los grandes acuerdos comerciales preferenciales pueden aumentar la eficiencia debido al incremento de la competencia y al aprovechamiento de economías de escala.}

\section{Conflictos comerciales}

Las guerras comerciales que podrían producirse entre grandes bloques son el caso extremo de un peligro más general de conflictos comerciales crecientes generados por los ACP. Incluso los ACP bilaterales pueden irritar a economías excluidas que se vean así discriminadas en mercados vecinos o de socios comerciales importantes. Los patrones " $H \& S$ " pueden avivar tensiones entre "hubs" que pugnan por la influencia hegemónica y entre "spokes" que se esfuerzan tanto por obtener los favores de aquéllos como posiciones de mercado entre sí. La desigual distribución de ganancias entre "hubs" y "spokes" es otra fuente potencial de tensiones o conflictos. 


\section{FACILITACIÓN DEL COMERCIO}

La cobertura de los ACP en el AsiaPacífico usualmente se extiende mucho más allá de la liberalización del comercio de bienes y servicios, y comprende medidas para facilitar ese comercio que incluyen procedimientos aduaneros y los regímenes de admisión transitoria pertinentes, estándares, compras estatales y, últimamente, comercio electrónico. Muchos incluyen reglas sobre salvaguardias, antidumping y medidas compensatorias; asimismo, varios comprenden la liberalización de las inversiones y mecanismos para liberalizar los mercados internos, especialmente en temas como la política de competencia, la propiedad intelectual y la armonización del tratamiento de los negocios y de la tributación. Algunos comprenden estándares laborales y ambientales, y varios detallan regímenes especiales para ciertos sectores. Todo lo anterior explica la creciente popularidad del término "closer economic partnership (CEP) " o asociación económica más estrecha, acuñado para describir acuerdos de tan amplio alcance. A menudo se alaba a los CEP porque van más allá de la OMC (o sea, porque son "WTO-plus"), pero hasta algunos de los CEP más avanzados pueden ser "WTO-minus" en algunas materias, por ejemplo, en lo relativo a la agricultura.

Cuando las barreras comerciales son relativamente bajas, la mejora de la facilitación del comercio puede acarrear beneficios tanto o más importantes que los atribuibles a la liberalización y, en consecuencia, ser más decisivos para tor- nar positivo el balance neto de los efectos de $\mathrm{CC}$ y de DC generados por el ACP, razón por lo cual algunos $C E P$ modernos del Asia-Pacífico hacen hincapié en la facilitación del comercio. Pero, aunque los beneficios resultantes para los miembros puedan ser sustanciales, ello no implica necesariamente mejoras en la situación de los no miembros. Al estimular el comercio y la inversión entre los miembros, las medidas de facilitación de un ACP pueden discriminar de hecho contra los no miembros, aun cuando en este caso se trate del resultado de una genuina reducción de los costos de transacción y no de una ventaja artificial en materia de costos debido a preferencias arancelarias.

\section{Cuando las barreras comerciales son relativamente bajas, la facilitación del comercio puede acarrear beneficios tanto 0 más importantes que los de la liberalización.}

\section{REGLAS DE ORIGEN}

Las reglas de origen (RO) son necesarias para garantizar la integridad de las preferencias acordadas, y asegurar que éstas sean gozadas únicamente por los beneficiarios deseados y en la medida deseada. En tal sentido, constituyen uno de los aspectos más importantes de un ACP. Pero en la práctica, las RO pueden ser diseñadas de manera de satisfacer también a una variada gama de otros propósitos, que van desde la facilitación del 
comercio en un extremo, hasta la neutralización proteccionista de la preferencia arancelaria acordada a productos sensibles, en el otro. Los ACP, incluyendo los suscritos entre miembros del APEC, pueden entonces contener una amplia gama de RO, desde relativamente simples hasta muy complejas.

El cumplimiento de las RO suele aumentar significativamente el costo de hacer negocios. Aunque la investigación empírica al respecto todavía es escasa, según primeras estimaciones, dichos costos representan entre 3\% y $5 \%$ del valor fob de los bienes exportados ${ }^{3}$. Mientras mayor sea la complejidad de las RO, mayor la probabilidad tanto de que aumenten los costos de su cumplimiento como de que den lugar a costosas disputas. El peso de todo esto puede ser insostenible para los exportadores pequeños o medianos. Las RO también pueden imponer costos de eficiencia a los miembros del $\mathrm{ACP}$, aunque este efecto sea poco entendido hasta el momento.

\section{El cumplimiento de las reglas de origen suele aumentar el costo de} hacer negocios.

Al proliferar los ACP, las RO de los nuevos pueden acarrear la consecuencia, quizás no deseada, de descomponer los vínculos de integración logrados mediante los ya existentes. Un fabricante que utiliza libremente insumos calificados como originarios en un ACP existente, puede verse en la necesidad de reducir su uso para poder cumplir con las diferentes RO de un nuevo ACP con otro socio comercial. Esto puede ser motivo de particular preocupación cuando se desarrollan configuraciones " $H \& S$ " que involucran a "spokes" ya integrados entre sí por ACP existentes.

\section{El "SPAGHETTI BOWL"}

La proliferación de ACP puede incrementar la probabilidad de incompatibilidad entre las reglas y estipulaciones pertinentes, lo que traería consigo todo un conjunto de complicaciones y costos adicionales, especialmente para las economías pertenecientes a varios ACP. La más obvia y quizás también la más importante fuente potencial de incompatibilidades son las RO, cuyos costos de cumplimiento de por sí significativos pueden acrecentarse para los exportadores de economías que son miembros de múltiples ACP cuyas RO son incompatibles entre sí. Otras fuentes potenciales de efectos similares son los estándares, los procedimientos aduaneros y los regímenes de admisión temporal respectivos.

De ahí que se haya planteado ${ }^{4}$ el problema del creciente número de reglas y

${ }^{3}$ Estevadeordal, A. y Suominen, K., "Rules of Origin: a World Map", trabajo presentado en el Seminario PECC/LAEBA sobre ACR en Perspectiva Comparada: América Latina y el Caribe y Asia-Pacífico, Banco Interamericano de Desarrollo, Washington, D.C., abril de 2003 (disponible en: www.pecc.org/trade).

${ }^{4}$ Bhagwati, J., Greenaway, D. y Panagariya, A., "Trading Preferentially: Theory and Policy", Economic Journal, Vol. 108, 1998; pp. 1128-1148. 
estipulaciones incompatibles que enfrentarían las economías a medida que suscriban más y más ACP, y que les encarecerían los costos de transacción en sus negocios de exportación e importación, efecto que ha sido denominado "spaghetti bowl". La magnitud del incremento de los costos de transacción resultante es un asunto empírico que debe ser investigado porque aún no se dispone de estimaciones confiables al respecto. Entre los miembros del APEC, economías ya involucradas desde hace cierto tiempo en múltiples ACP, como Chile, México y Singapur, pueden servir de "laboratorios" útiles para investigar este tema. De verificarse la existencia de crecientes costos de transacción debido al efecto "spaghetti bowl", éste iría contra los objetivos del APEC en materia de facilitación del comercio, expresados en la Declaración de Bogor y más recientemente en el Acuerdo de Shanghai.

\section{La magnitud del incremento de los costos de transacción es un asunto empírico que hay que investigar.}

\section{Atractivo de los ACP}

La popularidad de los ACP es evidencia clara de que los gobiernos los consideran ventajosos, poruna o varias de las razones que se indican a continuación:

- La posibilidad de avanzar más y más rápidamente con sólo un grupo de socios comerciales y no con todos los partícipes de las negociaciones multilaterales;

- Como los ACP ofrecen oportunidades de exportación identificables con mayor precisión, son más fácilmente aceptables por las fuerzas políticas nacionales;

- Los ACP pueden diseñarse a la medida de las circunstancias específicas de los miembros;

- A diferencia del APEC, los ACP establecen compromisos vinculantes $\mathrm{y}$ resultantes de la negociación de concesiones recíprocas;

- Los ACP proporcionan mecanismos para discutir y resolver temas difíciles relacionados con el comercio y la inversión bilaterales;

- Los ACP pueden servir de "campo de entrenamiento" para la liberalización unilateral y multilateral, en el cual gobiernos y empresas aprenden a adaptarse a la mayor competencia resultante de la liberalización;

- Los ACP pueden contribuir al desarrollo económico, al proporcionar canales para la provisión de bienes públicos regionales;

- La negociación e implementación de ACP puede ser de utilidad para evitar la reversión de reformas económicas;

- La negociación e implementación de ACP puede ser de utilidad para el logro de objetivos de política exterior y de seguridad.

En un orden de ideas menos positivo, el atractivo de los ACP también puede deberse a la posibilidad que ofrecen de excluir de la negociación con algunos 
socios comerciales ciertos temas sensibles que tendrían que estar incluidos en una negociación multilateral.

\section{Uno de los motivos para tratar de llegar a acuerdos preferenciales es asegurarse mercados de exportación vulnerables.}

Las motivaciones que explican los ACP pueden variar según sean norte-norte, norte-sur, o sur-sur. Una motivación importante para los primeros suelen ser los beneficios potenciales debidos a las economías de escala y al aumento de la competencia ligados al comercio intraindustrial en mercados tecnológicamente dinámicos. Similar motivación puede conducir también a acuerdos norte-sur, aunque es probable que los mayores beneficios potenciales de estos se vinculen con el comercio interindustrial guiado por diferencias en materia de ventajas comparativas convencionales. Otra motivación para que las economías en desarrollo busquen este tipo de acuerdos consiste en asegurarse vitales mercados de exportación percibidos como vulnerables. Mientras que esas economías suelen establecer entre sí acuerdos sur-sur a fin de desarrollar mercados para sus exportaciones de productos con valor agregado, cuando éstas encuentran dificultades para penetrar mercados de países desarrollados debido, entre otros factores, a los bien conocidos fenómenos de picos y escalonamientos arancelarios.

\section{EFECTOS ECONÓMICOS DE ENFOQUES ALTERNATIVOS DE LIBERALIZACIÓN REGIONAL}

A la luz tanto de la evidencia proporcionada por unas cuarenta investigaciones empíricas basadas en modelos de equilibrio general calculable (o modelos $C G E$, por sus siglas en inglés) ${ }^{5}$ como de la lógica económica, a continuación se presenta un análisis comparativo de los distintos enfoques de liberalización regional adoptados por miembros del APEC, cuya conclusión central confirma la sabiduría del enfoque de éste. En efecto, los resultados de estas investigaciones muestran que tanto colectiva como individualmente la liberalización comercial a nivel del APEC en su conjunto puede producir beneficios mayores ${ }^{6}$ que cualquiera de los modelos de liberalización preferencial entre grupos de economías miembros del APEC. Esta conclusión es válida a la vez cuando el modelo general de liberalización es no discriminatorio, y cuando éste es preferencial, aunque una y otra moda-

\footnotetext{
5 "Summary of Results of CGE Analyses of Asia-Pacific RTAs", apéndice de "Asia-Pacific RTAs as Avenues to Achieving the Bogor Goals: Analysis and Ways Forward", documento presentado por el PECC al APEC SOM Trade Policy Dialogue on RTAs/FTAs, Khon Kaen, Tailandia, 27 de mayo de 2003 (documento APEC que lleva la signatura 2003/SOM II/RTAs/FTAs/012).

${ }^{6}$ Los resultados que arrojan los modelos $C G E$ para las distintas economías examinadas suelen compararse en función de variaciones porcentuales del bienestar económico. Es en tal sentido, entonces, que en esta sección se habla de beneficios (o pérdidas) mayores o menores, o de efectos grandes o pequeños.
} 
lidad repercute de manera diferente en el resto del mundo.

Tal conclusión se sustenta en una convincente lógica económica. La desviación de comercio será menor cuanto mayor sea la proporción de los flujos comerciales de los miembros abarcada por el acuerdo comercial. La región del APEC representa $70 \%$ o más de los flujos comerciales de sus miembros, e incluye la mayor parte de sus respectivos comercios bilaterales. Esto, unido al hecho de que el APEC incluye a economías en diferentes estadios de desarrollo, hace probable que la mayor parte de las importaciones de sus miembros comprendidas por una liberalización a escala del APEC en su conjunto provenga de proveedores competitivos en el plano internacional. Asimismo, puede ser que las medidas de facilitación del comercio que abarquen todo el APEC acarreen beneficios mayores a sus miembros que aquéllas de menor alcance. Por todo esto, cuando se compara la liberalización al nivel de APEC en su conjunto con la liberalización preferencial entre grupos de sus miembros, se comprueba que la primera asegura que todos los miembros tengan acceso a los mercados más dinámicos en los mejores términos posibles y genera mayores estímulos al crecimiento económico que la segunda.

Entre las distintas modalidades de liberalización preferencial entre grupos de economías de APEC, respecto de los ACP bilaterales entre economías pequeñas o medianas, no sorprende que tanto los be- neficios para los respectivos pares de miembros como los efectos negativos sobre los no miembros sean reducidos, puesto que los flujos comerciales abarcados por este tipo de ACP son relativamente pequeños. Sin embargo, estos efectos negativos pueden bastar para perjudicar a economías vecinas o que comercian intensamente con alguno de los miembros, los que podrían responder suscribiendo un ACP que les permita neutralizar el daño. La proliferación de ACP es en parte resultante de esta variante del efecto "dominó" ".

\section{Un acuerdo preferencial entre} una economía pequeña o mediana y una grande posiblemente aumentará más el bienestar de la primera que de la segunda.

Cuando los ACP bilaterales comprenden una economía pequeña o mediana y una economía grande, tampoco sorprende que la mejora del bienestar económico sea relativamente grande para la primera y relativamente reducida para la segunda, ya que la proporción del comercio abarcada por el ACP es mucho mayor en el primero que en el segundo caso. Sin embargo, este tipo de ACP bilateral puede acarrear significativos efectos negativos para los no miembros que sean vecinos o competidores de la economía miembro pequeña o mediana, que se verán obligados a suscribir su propio ACP con la

${ }^{7}$ Baldwin identificó el "efecto dominó" al señalar que, en los casos en que un bloque comercial se establece o progresa significativamente, surgen incentivos para que no miembros procuren integrarse a ese ACP, a fin de participar de sus beneficios y evitar los costos de permanecer excluidos. 
misma economía grande a fin de neutralizar tales efectos y lograr para sí los beneficios consiguientes. Esta versión del efecto "dominó", que refleja mejor la definición de Baldwin, es una poderosa propagadora del modelo " $H \& S$ " de ACP y, en los casos en que se combina con la otra variante antes mencionada, también da lugar a los " $H \& S$ secundarios".

Es ilustrativo comparar los efectos de un solo ACP bilateral entre un " $h u b$ " y un "spoke" con los que se producen cuando varios "spokes" logran simultáneamente suscribir ACP con el mismo " $h u b$ ". Un estudio reciente ${ }^{8}$ muestra que mientras que en el primer caso se producen los resultados típicos, en que el "spoke" se beneficia relativamente más que el " $h u b$ ", la situación se revierte en el segundo, en que son más significativos los beneficios para el " $h u b$ " y mucho menores aquellos de los "spokes". Así pues, al agregar nuevos "spokes" a un modelo " $H \& S$ " tienden a debilitarse las ventajas económicas de los "spokes" existentes. Los estudios también muestran que algo similar tiende a ocurrir respecto de los beneficios de los miembros existentes de un ACP, particularmente de los más pequeños, cuando se incorporan nuevos miembros al acuerdo.

Sin embargo, algunos análisis de los dos ALC propuestos, China-ASEAN y Japón-ASEAN, indican que estos acarrearían sustanciales beneficios a los miembros de ASEAN, pero que generalmente serían menores que los de un ALC "ASEAN + 3" para dichos miembros. La ventaja adicional de un ALC "ASEAN + 3" serían los beneficios que aportaría a las dos economías del noreste asiático, que quedarían excluidas en cada ALC "ASEAN + 1". Quizás sea aún más interesante que algunos análisis indiquen que incluso el miembro del noreste asiático de cada ALC "ASEAN + 1" obtendría mayores beneficios bajo un ALC "ASEAN + 3".

\section{Los acuerdos preferenciales pueden incubar tensiones y conflictos comerciales.}

Al mismo tiempo, cualquiera de los ACP mencionados en el párrafo precedente que suscriban miembros del ASEAN ocasionan pérdidas mayores a las economías excluidas que los ACP de menor alcance. Conforme aumenta el tamaño del ACP, crecen las pérdidas de los no miembros y éstas se concentran en un número progresivamente menor de economías que comercian intensamente con los miembros del ACP y que son miembros del APEC. Más específicamente, un ALC "ASEAN + 3", que entre los mencionados en el párrafo anterior sería el que más beneficiará a los miembros, sería también el que mayores pérdidas acarreará a las tres economías excluidas del Pacífico occidental: Australia, Nueva Zelandia y China Taipei. Una ampliación de tal ALC que incluyera a estas tres economías convertiría sus pérdidas en sustanciales ganan-

\footnotetext{
${ }^{8}$ Gilbert, J., "CGE Simulation of US Bilateral Free Trade Agreements", documento de base para la conferencia sobre "Free Trade Agreements and US Trade Policy", Institute for International Economics, Washington, D.C., 7-8 de mayo de 2003.
} 
cias, y acarrearía a la mayoría de los demás miembros beneficios iguales o incluso mayores que si no se ampliara.

Es importante resaltar las principales conclusiones del análisis comparativo presentado en esta sección. Los ACP bilaterales o plurilaterales entre grupos de economías del APEC probablemente producen beneficios económicos para sus miembros y, en tal medida, la proliferación observada puede considerarse como un paso hacia el logro de los beneficios que llevarían consigo los objetivos del APEC. Sin embargo, al mismo tiempo, esos ACP tienden a ocasionar pérdidas a los no miembros y, por ello, incuban tensiones y conflictos comerciales. Los ACP de mayor alcance entre economías del APEC probablemente acarreen mayores beneficios económicos a sus miembros, $y$ pueden considerarse un avance en el sentido de una liberalización a escala de todo el APEC; pero, al mismo tiempo, también es probable que estos ACP incrementen las pérdidas de los no miembros y, por ello, sean fuentes potenciales de aún mayores tensiones y conflictos comerciales.

En cambio, la aplicación de un enfoque amplio de liberalización que abarque al APEC en su conjunto aportaría más beneficios económicos que cualquiera de las opciones anteriores, y eliminaría las pérdidas que éstas imponen a las economías excluidas. Es decir, la reciente proliferación de ACP entre economías del APEC conlleva ventajas para los miembros que explican la popularidad de que gozan actualmente en la región, pero la comparación de los beneficios y costos no sólo no invalida sino que, por el contrario, confirma la clarividencia de los objetivos del APEC y la pertinencia de su enfoque de liberalización regional expresados en la Declaración de Bogor.

\section{Cuando reúnen las condiciones apropiadas, los acuerdos comerciales preferenciales pueden ser un mecanismo constructivo.}

El problema consiste, entonces, en cómo las economías de APEC podrían hacer la transición de la proliferación de ACP hacia el cumplimiento de las metas de Bogor. Este problema es análogo al ampliamente debatido acerca de cómo afecta la liberalización preferencial las perspectivas de la liberalización multilateral, que ha dado lugar a una extensa literatura sobre si los ACP son "building blocks" o "stumbling blocks" para el logro del libre comercio mundial. Anticipando las conclusiones de las secciones que siguen, la respuesta al problema frente a los objetivos de APEC es la misma que frente al libre comercio mundial, esto es, que los ACP pueden ser "building blocks" en la medida en que reúnan las condiciones apropiadas. La tarea clave consiste, entonces, en identificar estas condiciones y velar por que sean cumplidas. 
¿ "Building Blocks" o "Stumbling Blocks" para los OBJetivos Del APEC?

Para la transición entre la proliferación de ACP y el libre comercio a escala del APEC en su conjunto o a escala global, habría cuatro grandes caminos, no excluyentes entre sí.

- Formar una red de ACP bilaterales que abarque todos los pares de economías. No obstante que algunas economías del APEC (tales como Chile, México y Singapur) se han propuesto suscribir ACP con todos sus socios comerciales más importantes, hay razones para dudar de que éste pueda ser un camino realista o eficiente hacia el libre comercio a escala más amplia. $\mathrm{Si}$, como se ha señalado ${ }^{9}$, para lograr el equivalente de un libre comercio multilateral mediante ACP bilaterales en un conjunto de " $n$ " países, se necesitarían [n(n-1)/2] ACP bilaterales, esto es $210 \mathrm{ACP}$ bilaterales para lograr el libre comercio en los 21 miembros del APEC y 19.900 para lograrlo en un universo compuesto aproximadamente por 200 economías. Es difícil creer que ello pueda ser factible, y es muy probable que no sea un camino eficiente, en especial si las estipulaciones de los múltiples ACP fueran incompatibles en numerosos aspectos.

- Ampliar gradualmente los ACP existentes. Comparado con el camino anterior, éste sería más práctico y eficiente, porque reduce el número de ACP y porque probablemente la incorporación de nuevos miembros al ACP exclusivamente en los temas de acceso a mercados significaría tener que negociar nuevos $\mathrm{ACP}$ bilaterales entre aquél y cada uno de los miembros existentes, lo que es poco probable que ocurra respecto de las normas generales del ACP, y reduce aunque no elimina el problema del "spaghetti bowl" de estipulaciones incompatibles.

\section{En la transición del libre comercio a escala APEC, habría cuatro grandes caminos.}

- Reunir o hacer converger los ACP existentes. Este camino tendría que emprenderse en un escenario de ACP múltiples a fin de que sea posible su transición hacia el libre comercio a escala de todo el APEC o a nivel global.

- Realizar una liberalización no discriminatoria (NMF), multilateral o unilateralmente, y proceder paralelamente a una liberalización preferencial, aunque a un ritmo menor, implicaría la convergencia de todas las economías hacia el libre comercio a escalas más amplias, incluso si los ACP como tales no convergieran entre sí.

Las evidentes dificultades que encierra cada uno de los tres primeros caminos sirven para subrayar la importancia decisiva de asegurar el objetivo último avan-

${ }^{9}$ Lloyd, P., "New Regionalism and New Bilateralism in the Asia Pacific", presentado en la reunión del Foro de Comercio del PECC, Lima, mayo de 2002 (disponible en: www.pecc.org/trade). 
zando al mismo tiempo por el cuarto camino y dilucidar la cuestión de los efectos positivos o negativos que pueden tener los ACP en la liberalización multilateral y unilateral y respecto del cumplimiento de las metas de Bogor.

INCENTIVOS PARA LA PROLIFERACIÓN, AMPLIACIÓN O FUSIÓN DE ACP

La "liberalización competitiva" es un concepto en boga ${ }^{10}$, muy similar al "efecto dominó" ya mencionado, en virtud de la cual la suscripción de ACP, particularmente cuando uno de sus miembros es una potencia económica, crea incentivos para que otros países procuren lo mismo, ya sea mediante nuevos ACP o incorporándose a los existentes. La motivación básica es la defensa de posiciones de mercado en los principales socios comerciales, que se ven amenazadas por los ACP suscritos por los competidores. Una motivación similar puede también conducir a que miembros de distintos ACP procuren reunirlos en uno solo. La "liberalización competitiva" busca expresamente sacar provecho de los efectos negativos de la discriminación inherente a los ACP, usándolos como mecanismo de presión para que otros países ingresen al campo de la liberalización preferencial $\mathrm{y}$, mediante este proceso, ejerzan más presiones por avanzar en la liberalización multilateral.
Lo que está ocurriendo en la región del Asia-Pacífico, en que una potencia económica como los Estados Unidos se muestra dispuesta a suscribir ACP bilaterales o plurilaterales, es prueba de lo que pueden reclamar a su favor los proponentes de la "liberalización competitiva"; y los ya mencionados análisis empíricos muestran que en estos casos el "efecto dominó" es bastante marcado. También pueden surgir propuestas de fusión de ACP existentes, como por ejemplo, de reunir el AFTA y el CER en un solo ACP. Aunque hasta hoy ha habido muy pocos indicios de que economías del APEC deseen incorporarse a ACP existentes, es probable que si el grupo "ASEAN + 3" suscribiera un ACP para el oriente asiático, otras economías del Pacífico occidental, como China Taipei, Australia y Nueva Zelandia, estarían deseosas de incorporarse a él.

\section{La liberalización competitiva es un concepto similar al efecto dominó.}

Está claro, entonces, que la "liberalización competitiva" efectivamente ejerce presión para que las economías excluidas pugnen por entrar en el campo de la liberalización preferencial. Menos claro es, empero, el incentivo que puedan tener los miembros de un ACP para admitir nuevos miembros, pues el análisis al respecto $^{11}$ señala que ese incentivo depen-

${ }^{10}$ Ver, por ejemplo, Bergstern C. Fred, "A Renaissance for US Trade Policy”, Foreign Affairs, Vol. 81, $\mathrm{N}^{\circ}$ 6, noviembre/diciembre 2002; pp. 86-98.

${ }^{11}$ Andriamananjara, S., "On the Relationship between Preferential Trading Arrangements and the Multilateral Trading System", presentado a la reunión del Foro de Comercio realizada en Washington, D.C., abril 2003 (disponible en: www.pecc.org/trade). 
de de la acción de dos efectos contrapuestos: por un lado, los beneficios que puede acarrear ampliar el tamaño del mercado resultante de la incorporación de nuevos miembros y, por el otro, las pérdidas que lleva consigo tal incorporación al reducirse las preferencias. El análisis revela que el primero de estos efectos tiende a ser dominante en las primeras etapas de la ampliación de un ACP, pero que en las siguientes generalmente prevalece el segundo, hasta que desaparece el incentivo a admitir nuevos miembros, lo que significa que el ACP no conduce por sí al libre comercio a escala global, o quizás tampoco a escala de todo el APEC.

\section{Para la economía, la gravitación del segundo efecto en las etapas finales del proceso puede ser mayor si las excluidas son pequeñas.}

En términos económicos, la gravitación del segundo efecto en las últimas etapas del proceso será aún mayor si las economías que siguen excluidas son pequeñas, de las cuales pueden obtenerse pocos beneficios como consecuencia de la ampliación del mercado. Pero ciertas cosas que suceden en la región del AsiaPacífico, por ejemplo la oposición de algunos miembros de ASEAN a que el $A F T A$ y el $C E R$ se fusionen en un solo ACP, o a que Australia y Nueva Zelandia puedan integrarse a un eventual ACP del este asiático, indican que no sólo razones económicas sino también políticas pueden obstaculizar la ampliación, fusión o convergencia de ACP. A la lista de obstá- culos no fáciles de superar, habría que agregar las divisiones y conflictos que puede generar el propio proceso de proliferación de ACP.

Adicionalmente, hay que tener en cuenta las connotaciones especiales que adquiere la estructura de incentivos cuando la "liberalización competitiva" opera por acción de un gran "hub" (por ejemplo, los Estados Unidos) mediante la suscripción de varios ACP bilaterales en forma simultánea o consecutiva. Para el " $h u b "$, hay mejoras en materia de bienestar económico agregado que lo incentivan a proceder así, pese a que algunas negociaciones con socios competitivos en determinados sectores sensibles podrían verse frustradas por la oposición de los respectivos grupos nacionales de intereses. Sin embargo, este escenario acarrea efectos inequívocamente negativos para las economías que primero suscribieron ACP con el " $h u b$ ", ya que los nuevos ACP de éste diluirán las preferencias que consiguieron sin proporcionarles ningún acceso adicional a mercados. Esas economías se verán entonces incentivadas a tratar de obstruir, o cuando menos a no propiciar, la negociación de nuevos ACP por parte del " $h u b$ ". En la región del AsiaPacífico hay indicios de este comportamiento.

\section{Efectos de los ACP en los \\ INCENTIVOS PARA LA LIBERALIZACIÓN MULTILATERAL}

El análisis económico aún no ha dado una respuesta concluyente a la pregunta 
de si los ACP incrementan o disminuyen los incentivos a la liberalización multilateral. La respuesta depende en parte de si dichos incentivos se miran desde la perspectiva de una economía en su conjunto o de las utilidades de las empresas que operan en ella. Si el nivel de bienestar económico agregado que generan los ACP existentes es menor que el de la liberalización multilateral, habrá incentivo para que la mayor parte de la población de al menos uno de los potenciales miembros del ACP rechace la opción de la liberalización preferencial y es partidaria de la multilateral.

\section{Aún no se dilucida si los acuerdos preferenciales aumentan 0 disminuyen los incentivos a la liberalización multilateral.}

Pero, al mismo tiempo, las utilidades que los ACP reportan a las respectivas empresas nacionales serán un incentivo para que presionen en favor de la opción preferencial y para que, una vez suscrito el ACP, se opongan a toda liberalización multilateral que pueda diluir las preferencias y reducir así la rentabilidad económica basada en éstas. Ello ocurrirá incluso en los casos de ACP que reducen el bienestar económico agregado de sus miembros debido a que predomina la desviación del comercio, ya que dicha rentabilidad empresarial probablemente será mayor mientras más comercio desvíe un
ACP. Sin embargo, casos como el del ANZCERTA demuestran que en algunos casos un ACP puede ayudar a que el sector empresarial sintonice con la necesidad de ajustarse a un contexto de liberalización comercial y, de este modo, abrir el camino para una posterior liberalización NMF amplia, realizada unilateralmente en los casos de Australia y Nueva Zelandia.

También es dudosa la validez del argumento que esgrimen quienes proponen la "liberalización competitiva", de que la formación o amenaza de formación de un gran ACP debilita la resistencia de otras economías a avanzar en negociaciones multilaterales. Por ejemplo, Bhagwati ${ }^{12}$ ha cuestionado la afirmación de Bergsten ${ }^{13}$ de que la potencial conversión del APEC en un mecanismo de liberalización eficaz sirvió para que la Unión Europea participara constructivamente en la conclusión exitosa de la Ronda Uruguay.

Respecto de otras formas en que la proliferación de ACP puede inhibir la liberalización no discriminatoria (unilateral o multilateral), una de ellas que ha sido poco examinada consiste en que las economías frenan esta última a fin de retener barreras comerciales para que las preferencias que pueden conceder resulten más apetitosas e incrementar así su poder de negociación y su atractivo como potenciales socios de ACP. Otra, frecuentemente mencionada, es la apreciable cantidad de recursos humanos e institucionales y de capital político que absorbe la nego-

${ }^{12}$ Bhagwati, J., "Dissent at APEC Meeting Cannot be Ignored”, Financial Times, 6 de diciembre de 1996.

${ }^{13}$ Bergsten, F., "Sunrise in Seattle", International Economics Insights, Vol. 5, № 1, Enero/Febrero 1994. 
ciación de ACP y que distrae de la negociación multilateral, lo que debilita la participación en esta última, especialmente de economías pequeñas en que dichos recursos son particularmente escasos.

Otro tema tiene que ver con la aparente estrategia de algunas economías (por ejemplo Chile, México y Singapur, entre los miembros del APEC) de suscribir ACP con todos sus principales socios comerciales, estrategia que, de aplicarse exitosamente, podría hacer que estas economías tuvieran escaso incentivo a participar activamente en las negociaciones multilaterales. Al mismo tiempo, empero, como hay temas comerciales que son de vital interés para esas y otras economías sobre los que sólo se puede avanzar en el plano de la OMC, es probable que continúen activamente involucradas en el proceso multilateral.

TRATAMIENTO DE SECTORES SENSIBLES

Se debate ampliamente si es legítimo o no que los ACP excluyan a sectores sensibles, debate que es típicamente conducido en función de si tal exclusión es compatible o no con las estipulaciones pertinentes del artículo XXIV del GATT. El análisis económico revela que dicha exclusión puede tener efectos negativos o positivos en el bienestar económico, según si el sector excluido es competitivo en uno solo de los miembros de un ACP bilateral, en cuyo caso disminuyen los beneficios por concepto de creación de comercio, o en ambos miembros, caso en que disminuyen las pérdidas por desviación de comercio.

\section{Se debate ampliamente si es legítimo o no que los acuerdos preferenciales excluyan sectores sensibles.}

Desde el punto de vista del debate sobre los "building blocks y los stumbling blocks", interesa saber si la exclusión de sectores sensibles en los ACP contribuye o no a la causa de la liberalización a escalas más amplias (APEC o global), ya que el peligro consiste en que la capacidad de hacer esa exclusión lleve a las economías que tienen varios sectores sensibles a preferir la liberalización preferencial a la no discriminatoria.

\section{INCOMPATIBILIDADES ENTRE DIVERSOS "MODELOS" DE ACP}

El desarrollo de varias modalidades de " $H \& S$ " puede avivar la competencia entre diferentes "modelos" de ACP promovidos por los respectivos "hubs". Además de los dos principales existentes, que propusieran los Estados Unidos y la Unión Europea, en la región del Asia-Pacífico podrían aparecer otros "modelos", de inspiración japonesa, china y coreana. Las diferencias entre los dos grandes "modelos" existentes suelen darse en temas en que los enfoques de los Estados Unidos y la UE compiten por prevalecer a nivel del sistema multilateral de comercio, con vistas a lo cual van acumulando fuerzas mediante la aceptación lograda a nivel de los ACP. Las diferencias más obvias radican en las $\mathrm{RO}$, pero también 
se dan en varios otros temas, incluso los muy controvertidos sobre estándares laborales y medioambientales.

La posibilidad de convergencia entre esos "modelos" probablemente depende de si la coincidencia en temas como los mencionados se logra o no en el plano multilateral. Pero el desarrollo de conjuntos de ACP basados en "modelos" incompatibles y quizás inconciliables puede inhibir tal convergencia. Al respecto, la propagación de "modelos" que contienen rasgos inaceptables a nivel de la OMC no sería un fenómeno positivo para el sistema multilateral de comercio. La identificación de estos rasgos no deseables es, entonces, una tarea importante si lo que se desea es que los ACP sean "building blocks" para el libre comercio global o a nivel del APEC. Un ejemplo claro son las muy restrictivas y complejas $\mathrm{RO}$ del NAFTA, que se están dando también en los ACP suscritos recientemente por los Estados Unidos. Otros rasgos controvertidos, como el tratamiento de sectores sensibles o los estándares laborales y medioambientales, debieran revisarse desde la misma perspectiva.

\section{Compatibilidad CON LA OMC}

Cada vez que surge un nuevo ACP, sus miembros sostienen que es compatible con las normas de la OMC. No hay duda alguna de que es importante que los ACP lo sean, pero también lo es reconocer que las normas que impone la OMC a los ACP son bastante débiles, debido a las bien conocidas imprecisiones y ambi- güedades del artículo XXIV del GATT y del artículo V del GATS, así como a la incapacidad de los miembros de la OMC de llegar a un acuerdo sobre la interpretación de estas disposiciones. Se sigue así careciendo de definiciones universalmente aceptadas sobre una serie de requisitos básicos como, por ejemplo, el relativo a que los ACP deben abarcar lo "sustancial del comercio" entre sus miembros. Por esta razón, no sorprende que el Comité sobre ACR de la OMC sólo haya podido llegar a un consenso sobre la compatibilidad con ésta tan sólo respecto de uno de los más de $100 \mathrm{ACP}$ sometidos a su examen, y es poco probable que lo logre en el futuro próximo respecto de algún otro ACP. En la práctica, entonces, cada miembro de la OMC goza de un amplísimo margen de libertad para interpretar a su manera los mencionados artículos.

\section{Los acuerdos comerciales preferenciales no recíprocos entre economías desarrolladas son inadmisibles.}

Esto no significa que en materia de ACP para la OMC cualquier cosa vale. Existe cierto entendimiento común sobre las disposiciones en cuestión que impide algunas prácticas al respecto. Por ejemplo, no son admisibles ACP no recíprocos entre economías desarrolladas, lo que descarta la sugerencia del $E P G$ de que las economías del APEC que son miembros de ACP amplíen voluntariamente las res- 
pectivas preferencias a las demás economías del foro. Tampoco es admisible que las economías desarrolladas participen en acuerdos que conceden preferencias parciales, salvo con arreglo a esquemas del tipo SGP a las que puedan acceder todas las economías en desarrollo y cumplir así con los criterios fijados en la Cláusula de Habilitación. Pese a entendimientos comunes como estos, sigue siendo deseable esclarecer y mejorar las normas de la OMC en materia de ACP. Esta aspiración consta en la Agenda sobre Desarrollo de Doha, pero las perspectivas de lograr mejoras significativas al respecto no son prometedoras.

\section{Es posible que los acuerdos preferenciales políticamente más difíciles de lograr sean los más beneficiosos en lo económico.}

Una de las implicaciones de lo anterior es que, tal como son, en la actualidad, las normas de los OMC sobre ACP no garantizan que los miembros de ACP entre economías del APEC adoptarán las medidas necesarias para asegurar que sus acuerdos contribuyan al logro de las metas de Bogor. Por ejemplo, estas normas no exigen la adopción de un enfoque amplio que permita que eventualmente todas las economías de APEC gocen de los beneficios resultantes de las concesiones negociadas con arreglo a estos ACP, ni exigen que los miembros limiten los efectos negativos de la DC resultante de sus ACP, por ejemplo, continuando paralela- mente su proceso de liberalización gradual no discriminatoria; ni exigen la adopción de medidas para que sus ACP minimicen los problemas potenciales del "spaghetti bowl" o de rasgos no deseables para el sistema mundial de comercio, como lo es, por ejemplo, el uso de RO innecesariamente restrictivas.

\section{CONSIDERACIONES POLÍTICAS}

La materialización de algunos de los grandes ACP proyectados en la región del Asia-Pacífico enfrenta problemas políticos. Tal vez no sea exagerado afirmar que los ACP más beneficiosos en términos económicos son precisamente los más difíciles de lograr en términos políticos. En cambio, ACP que acarreen menos beneficios económicos llegan a concretarse, entre otras razones, porque políticamente es más fácil concertarlos. Todo esto puede estar generando divisiones quizás difíciles de superar entre las economías.

\section{CONCLUSIÓN: EL CAMINO HACIA LAS METAS DE BOGOR MEDIANTE LA} SUSCRIPCIÓN DE ACP NO ES AUTOMÁTICO

El análisis precedente no proporciona una respuesta concluyente a la cuestión de si los ACP son "building blocks" o "stumbling blocks" para el logro de las metas de Bogor, pero sí muestra las diversas maneras en que la descoordinada proliferación de ACP en la región del Asia-Pacífico puede frustrar esas metas. Una acción deliberadamente coordinada 
entre las economías del APEC es en consecuencia necesaria para asegurar que dicha proliferación contribuya a un proceso de liberalización que termine por abarcar toda la región. Para que sea posible una acción de esta naturaleza, se requiere que las economías de APEC adopten y apliquen en la región un "entendimiento común" sobre los ACP. Con el ánimo de contribuir a tal cometido, el Foro de Comercio del PECC ha elaborado propuestas para dicho "entendimiento común" a nivel del APEC ${ }^{14}$, cuyo propósito es asegurar que los ACP de las economías de la región sean "building blocks" para el logro de las metas de Bogor.

${ }^{14}$ PECC Trade Forum, "Proposals for an APEC Common Understanding on RTAs", PECC, septiembre 2003 (disponible en: www.pecc.org/trade). 